\title{
ASTROMETRY OF MILLIJANSKY SOURCES USING A PHASE REFERENCE VLBI TECHNIQUE
}

\author{
Jean-François Lestrade \\ Bureau des Longitudes, Paris, France \\ and Jet Propulsion Laboratory \\ Alan E. E. Rogers and Arthur E. Niell \\ Haystack Observatory, Westford, MA 01886 \\ Robert A. Preston \\ Jet Propulsion Laboratory, Pasadena, CA 91109
}

\begin{abstract}
Atmospheric fluctuations and hydrogen maser clock instabilities limit the coherence time $\left(T_{c o h}\right)$ and hence the signal-to-noise ratio of VLBI data $\left(S N R \propto \sqrt{T}_{\text {coh }}\right)$, to a few minutes at $\mathrm{GHz}$ frequencies. However, $T_{\text {coh }}$ can be increased up to many hours if a phase reference is established throughout the experiment by using a strong source observed alternatively with the program source. Weak celestial radio sources with flux densities in the milliJansky range at centimeter wavelengths, such as radio stars, fast pulsars and faint components of extragalactic sources, can then be reliably detected by this VLBI technique, simultaneously providing accurate astrometry relative to the reference source.
\end{abstract}

An arbitrarily small signal from the program source can be tracked by the VLBI correlator over successive scans of the experiment if fringe search is replaced by a fringe filter. The fringe filter is implemented by restricting the "search" to a fixed delay and fringe rate (i.e. a zero width window search) determined from the reference source fringe search for each scan. For the program source signal to be within the fringe filter passband, the uncertainty in the program source delay and rate must be smaller than $1 / b$ sec in delay and $1 / T_{\text {int }} \mathrm{Hz}$ in fringe rate or, for example, $<35$ nanosec and $<16 \mathrm{mHz}$ for a bandwidth $b=28 \mathrm{MHz}$ (Mark III mode B) and a scan integration time $T_{\text {int }}=60 \mathrm{sec}$. Such small uncertainty is not usually achieved in VLBI because the clock offset and rate are larger and are a priori unknown. However, if the clock parameters are first derived from the reference source observed throughout the experiment, then the fringe processing parameters for the program source scans can be properly set.

As a second step of the procedure, all the "noisy" complex visibilities of the program source must be coherently added together to built up the final $S N R$. This coherent addition can be carried out with the differenced phases between the program and reference sources computed by a precise astrometric model. If the uncertain parameter of this model is only the position of the program source, a grid of possible positions on the sky can be searched to obtain the position which optimizes the coherent addition.

The stellar system Algol and the nearby reference source MW 0309+41, separated by 70 arcminutes, were observed for 6 hours at $5 \mathrm{GHz}$ at both left and right circular polarizations on 1984 October 11. The total flux density of Algol was approximately 4 milliJansky or about $1 / 6$ the detection threshold provided by each 2 -minute scan on the 
two baselines between Greenbank and the VLA (GY) and between Owens Valley and the VLA (OY). The coherent addition was carried out over all the scans of Algol acquired on the two baselines in both senses of circular polarization. The resulting effective coherent integration time is $\mathbf{4 . 2}$ hours, providing a $\mathbf{1 0}$-fold increase in sensitivity compared to the original 2-minute scan. The coherent addition of all the complex visibilities over a grid of possible positions of Algol yields the "dirty" map in Fig. 1. High side lobes are apparent in this map because the u-v coverage (Fig. 2) is poor. However, it is sufficient to unambiguously locate the peak of the map to provide the astrometric position of Algol.

Several data sets were formed from the two baselines GY and OY and the two senses of circular polarization $L$ and $R$. There are 4 independent estimates of the position of Algol relative to $\mathrm{MW} 0309+41$ (Table 1). Uncertainties in station locations, earth orientation parameters, ionospheric and tropospheric effects and errors in the extrapolation of the reference source phase, can cause systematic errors as large as 1.5 milliarcsecond according to our analysis. The stochastic errors (angular resolution/SNR) are 0.8 and 1.2 milliarcsecond in $\alpha$ and $\delta$, respectively, for an SNR of 6 . Hence, the spread of 1.5 milliarcsecond in $\alpha$ and 1.7 milliarcsecond in $\delta$ found in Table 1 is consistent with the expected precision.

\begin{tabular}{|c|c|c|c|c|}
\hline $\begin{array}{l}\text { Baseline/ } \\
\text { Polarization }\end{array}$ & $\begin{array}{c}T_{\text {coh }} \\
\text { hours }\end{array}$ & $\begin{array}{l}\Delta \alpha \\
4^{m}\end{array}$ & $\begin{array}{l}\Delta \delta \\
22^{\prime}\end{array}$ & SNR \\
\hline OY/L & 1.1 & $51^{\circ} .83081$ & $40 " .8348$ & 6 \\
\hline OY/R & 1.0 & $51^{\circ} .83079$ & $40 " .8350$ & 7 \\
\hline OY/I & 2.1 & $51^{8} .83079$ & $40 " .8348$ & 9 \\
\hline GY/L & 1.0 & $51^{\circ} .83071$ & $40 " .8360$ & 6 \\
\hline GY/R & 1.1 & $51^{\circ} .83073$ & $40 " .8365$ & 6 \\
\hline GY/I & 2.1 & $51^{\circ} .83078$ & $40 " .8360$ & 9 \\
\hline $\mathrm{OY}+\mathrm{GY} / \mathrm{L}$ & 2.1 & $51^{\circ} .83076$ & $40 " .8360$ & 8 \\
\hline $\mathrm{OY}+\mathrm{GY} / \mathrm{R}$ & 2.1 & $51^{\circ} .83076$ & $40 " .8362$ & 9 \\
\hline $\mathrm{OY}+\mathrm{GY} / \mathrm{I}$ & 4.2 & $51^{\circ} .83076$ & $40 " .8360$ & 12 \\
\hline
\end{tabular}

Table 1: VLBI relative position of MW 0309+41 minus Algol. The symbol $I$ is for the sum of the LCP and RCP data. If the reference source MW 0309+41 is at $\alpha=3^{h} 13^{m} 1^{\bullet} .9615$ and $\delta=41^{\circ} 20^{\prime} 1^{n} .202$ then Algol is at $\alpha=3^{h} 8^{m} 10^{s} .1307 \pm 0^{s} .0001$ and $\delta=40^{\circ} 57^{\prime} 20^{\prime \prime} .366 \pm 0^{\prime \prime} .0017$. The positions are in the J2000.0 system and neither the parallax nor the proper motion effects have been removed.

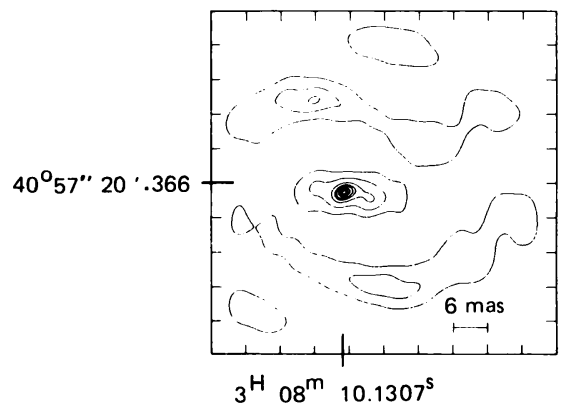

Figure 1: Coherent addition of visibilities over a grid of possible positions for Algol. Contour levels: $10,50,75,85,90,95,99 \%$ peak
(MILLION WAVELENGTHS, $5000 \mathrm{MHz}$ )

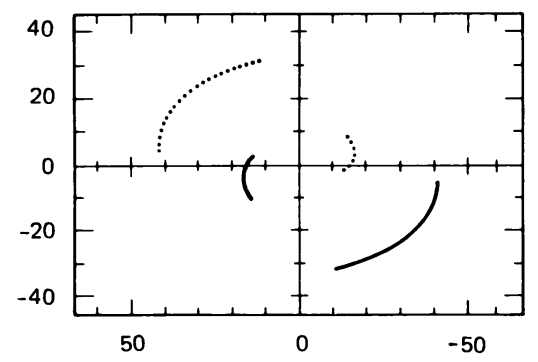

Figure 2: u-v coverage of the VLBI observation of Algol and MW 0309+41 